14.03

\title{
Фокусированный ультразвук высокой интенсивности: тепловой нагрев и разрушение биологической ткани
}

\author{
(С Т.А. Андреева, А.Е. Беркович, Н.Ю. Быков, Ф.В. Козырев, А.Я. Лукин \\ Санкт-Петербургский политехнический университет Петра Великого, \\ 195251 Санкт-Петербург, Россия \\ ฯ e-mail: bykov_nyu@spbstu.ru, nbykov2006@yandex.ru
}

Поступило в Редакцию 17 февраля 2020 г.

В окончательной редакции 17 февраля 2020 г.

Принято к публикации 17 фревраля 2020 г.

Представлены результаты численного исследования процессов нагрева и разрушения биологической ткани под действием серии импульсов фокусированного ультразвука (программы обработки опухоли), генерируемого многоэлементным излучателем. Рассмотрены программы облучения, отличающиеся пространственной локализацией фокальных точек в ткани (спираль Архимеда, „квадратная““ спираль), числом импульсов в серии, величиной задержки между импульсами. Установлено существенное влияние параметров серии импульсов и учета процесса перфузии на границы области теплового поражения ткани в рассмотренном диапазоне умеренных интенсивностей излучения. Показано, что с течением времени процесс распространения тепла в ткани приобретает квазисферический характер и слабо зависит от вида программы облучения. Обсуждается возможность использования данного свойства процесса для оптимизации протоколов медицинских процедур, связанных с применением фокусированного ультразвука.

Ключевые слова: фокусированный ультразвук, абляция биоткани, объемное энерговыделение, уравнение теплопроводности.

DOI: $10.21883 / J T F .2020 .09 .49685 .54-20$

\section{Введение}

В последнее десятилетие наряду с традиционными хирургическими методами удаления опухолей различной природы все большее распространение находят высокотехнологичные методы лечения, такие как фокусированный ультразвук высокой интенсивности (high-intensity focused ultrasound, HIFU) [1-5]. Технология HIFU является одной из перспективных неинвазивных методик лечения рака молочной, предстательной, щитовидной желез, печени и почек. Она предусматривает воздействие серии импульсов ультразвукового излучения на биологическую ткань, приводящее к некрозу опухоли. Процедура HIFU требует планирования параметров единичных импульсов в серии (интенсивности излучения, длительности импульса), задания временной последовательности включения импульсов и пространственной локализации точек фокуса отдельных импульсов в ткани. Правильный выбор параметров определяет качество терапии, результатом которой должен являться некроз всего объема опухоли. Контроль температурного режима в ходе HIFU-воздействия важен для минимизации как возможных повреждений здоровой ткани, так и неблагоприятных эффектов, таких как разрыв опухоли с последующим метастазированием через кровоток.

Настоящая работа посвящена теоретическому исследованию воздействия импульсов фокусированного ультразвука на заданный объем биологической ткани с параметрами, характерными для жировых тканей грудной клетки. Моделирование процедуры HIFU в общем случае предполагает математическое описание согласованных процессов распространения ультразвуковых волн в среде и нагрева ткани, а при определенных условиях и сопутствующих процессов (кавитации, химических трансформаций). Как правило, задача о распространении ультразвука в среде и задача расчета теплового поля с определением зоны термического поражения (абляции) решаются независимо [6-10]. По параметрам ультразвукового поля определяется мощность тепловых источников, используемая в дальнейшем при решении уравнения теплопроводности.

В настоящей работе рассматриваются умеренные интенсивности ультразвукового излучения порядка $100 \mathrm{~W} / \mathrm{cm}^{2}$ (в точке фокуса). С одной стороны, выбор такого диапазона обусловлен рассмотрением именно HIFU-воздействия на ткань, так как интенсивности менее $5 \mathrm{~W} / \mathrm{cm}^{2}$ обычно классифицируются как ультразвук низкой интенсивности [11]. С другой стороны, при больших интенсивностях излучения в среде становятся существенными нелинейные эффекты [2,5,12,13], а также эффекты кавитации [1-3]. Единое мнение научного сообщества о возможности использования кавитации, сопровождающей при определенных условиях HIFU-терапию, в настоящий момент отсутствует [3]. Кавитационный процесс усиливает поражающий эффект HIFU-воздействия, но является стохастическим, его наличие может привести к непредсказуемым размерам области разрушения, а также к распространению частей разрушенных клеток по всему организму больного. С точки зрения моделирования процедуры $\mathrm{HIFU}$, учет нелинейных эффек- 

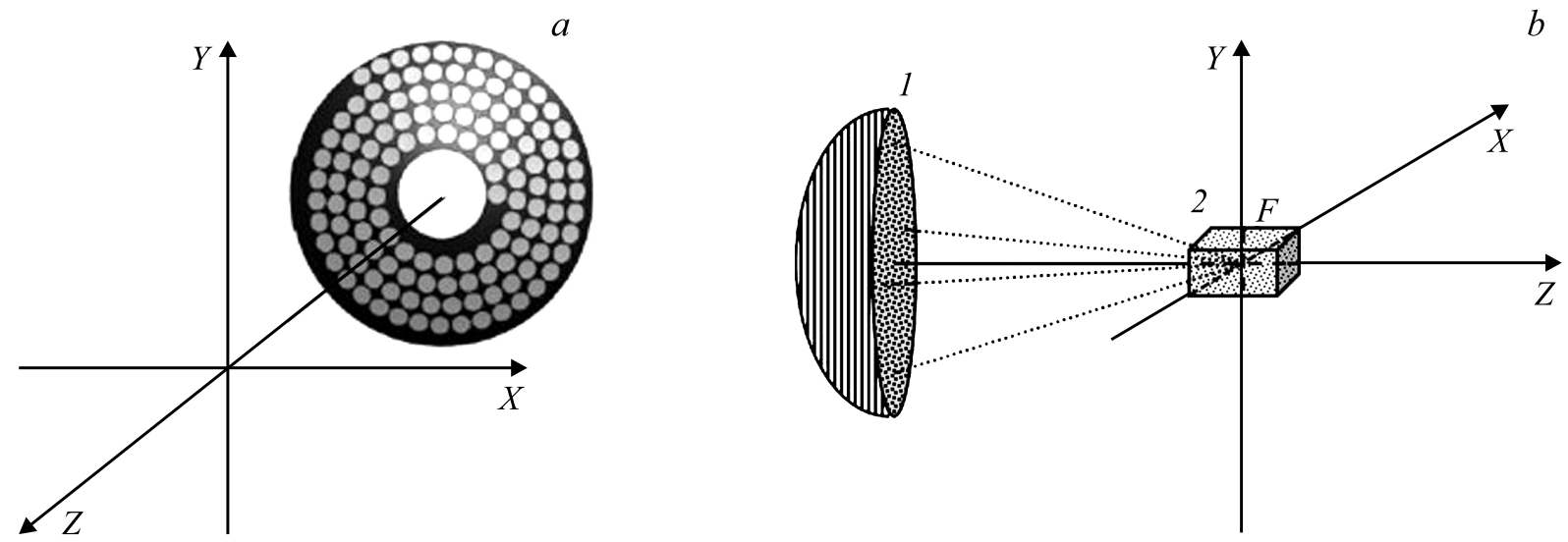

Рис. 1. 128-элементный излучатель $(a)$ и схема расчета $(b): 1-$ излучатель, 2 - прогреваемый объем ткани, $F-$ геометрический фокус излучателя.

тов в расчете требует достаточно трудоемкого решения нелинейных уравнений эволюционного типа [6,7], или решения уравнений Навье-Стокса для сжимаемой среды [14]. В настоящей работе используются результаты традиционного подхода к определению интенсивности звукового поля, основанного на вычислении интеграла Рэлея [8-10]. Моделирование распространения тепла базируется на численном решении уравнения теплопроводности [15], описывающего нестационарный пространственный процесс.

Стремительное развитие компьютерных технологий в 80-90x годах прошлого века способствовало активному применению численных методов для решения задач прогрева биоткани фокусированным ультразвуком. Среди большого числа работ по данному направлению (в том числе упомянутых ранее) необходимо отметить работы [6,10,16-18]. В работах [10,18] проведено численное исследование влияния длительности и интенсивности одиночного импульса, частоты излучателя, скорости перфузии на размеры области поражения. Работа [16] посвящена анализу воздействия серии из четырех последовательно включаемых импульсов на биологическую ткань. Процессы нагрева и разрушения ткани под действием режима одновременного формирования и перемещения девяти фокусов, создаваемых мощной акустической решеткой со случайным и регулярным расположением 256 элементов, изучались в работе [17]. В работе [6] представлены результаты моделирования процесса объемной тепловой абляции ткани говяжьей печени exvivo, создаваемой с помощью многоэлементного ультразвукового излучателя. В большей части работ, посвященных расчетам процессов теплопереноса, моделируется относительно небольшой временной диапазон распространения тепла от нескольких секунд до десятков секунд, основное внимание уделяется эволюции теплового состояния ткани и анализу конечных распределений тепловой дозы.

Настоящая работа является развитием работы [18], посвященной анализу воздействия на ткань одиночного импульса. Цель работы - численное исследование воздействия программы обработки, состоящей из нескольких десятков импульсов на процессы нагрева и разрушения биоткани. Существенное внимание уделяется анализу особенностей процесса распространения тепла для больших (по отношению ко времени воздействия) времен наблюдения (до $310 \mathrm{~s}$ ) и обсуждению использования результатов для медицинских приложений.

\section{1. Постановка задачи}

В качестве источника ультразвукового излучения в работе рассматривается многоэлементный излучатель фирмы Sonic Concepts (рис. 1), технические характеристики которого [19]: 128 излучающих элементов, расположенных по спирали Архимеда на вогнутой сферической поверхности, внешний диаметр излучателя $150 \mathrm{~mm}$, внутренний - $44 \mathrm{~mm}$, радиус кривизны $150 \mathrm{~mm}$, рабочая частота $2 \mathrm{MHz}$, максимальная интенсивность звукового поля в фокусе устройства до $80 \mathrm{~kW} / \mathrm{cm}^{2}$. Излучающие элементы имеют диаметр $10.15 \mathrm{~mm}$. Преимуществом многоэлементного излучателя является возможность электронного перемещения фокуса без необходимости физического перемещения самого устройства в пространстве.

Рассматривается нестационарный процесс нагрева биоткани под действием серии импульсов фокусированного излучения от источника ультразвука (рис. 1). Используется декартова система координат, центр координат совпадает с геометрическим фокусом излучателя, ось $Z$ совпадает с осью излучателя HIFU.

Процесс описывается уравнением теплопроводности [15]

$$
c \rho \frac{\partial T}{\partial t}=\operatorname{div}(\lambda \operatorname{grad} T)+\sum Q,
$$

где $T-$ температура, $t-$ время, $c, \lambda, \rho-$ теплоемкость, коэффициент теплопроводности и плотность среды соответственно, параметр $\sum Q-$ определяет удельную мощность внутренних источников теплоты. 
В случае постоянного коэффициента теплопроводности и рассмотрении двух источников тепла, связанных с процессами метаболизма и перфузии, данное уравнение в литературе получило название „биотеплопроводности“ (Bio-Heat Transfer Equation) [20-22].

Источником теплоты является энергия ультразвукового излучения. В окрестности фокальной точки волну можно считать квазиплоской и полагать мощность тепловыделения равной [2]

$$
Q_{H}=2 \alpha I
$$

где $I$ - интенсивность ультразвукового излучения в ткани, $\alpha-$ коэффициент поглощения.

Во время действия одиночного импульса или импульса в серии поле интенсивности ультразвукового излучения $Q_{H}\left(X, Y, Z, t_{i}<t \leq t_{i}+t_{H}\right)\left(t_{i}-\right.$ время начала действия импульса в серии, $t_{H}$ - длительность одиночного импульса) для рассматриваемого многоэлементного излучателя определяется согласно аппроксимации, предложенной в [18]. Данная аппроксимация основывается на решении интеграла Рэлея [23] и может использоваться для малых и умеренных интенсивностей излучения.

В уравнении (1) возможен также учет процесса перфузии, предполагающий введение дополнительного слагаемого в правую часть уравнения

$$
Q_{p}=-c_{b} W_{b}\left(T-T_{b}\right),
$$

где $c_{b}, W_{b}, T_{b}$ - теплоемкость, величина объемного расхода и температура крови соответственно [20-22].

Используемые в работе теплофизические параметры $c=2348 \mathrm{~J} /(\mathrm{kg} \cdot \mathrm{K}), \lambda=0.21 \mathrm{~W} /(\mathrm{m} \cdot \mathrm{K}), \rho=911 \mathrm{~kg} / \mathrm{m}^{3}$ соответствуют параметрам жировой ткани грудной клетки [24]. С учетом имеющегося в литературе разброса данных по коэффициенту поглощения в работе рассмотрены два значения коэффициента: $\alpha_{\text {ref }}=7.25 \mathrm{~m}^{-1}$ для частоты излучения $f_{\text {ref }}=1 \mathrm{MHz}[25]$ (зависимость от частоты излучателя $f$ предполагается линейной, для излучателя с частотой $\left.2 \mathrm{MHz} \alpha=14.5 \mathrm{~m}^{-1}\right)$ и $\alpha=9.25 \mathrm{~m}^{-1}$ (соответствует $f=2 \mathrm{MHz}$ ) [24]. Параметры кровотока: $c_{b}=3617 \mathrm{~J} /(\mathrm{kg} \cdot \mathrm{K}), \quad W_{b}=0.746 \mathrm{~kg} /\left(\mathrm{m}^{3} \cdot \mathrm{s}\right) \quad[24]$, $T_{b}=36.5^{\circ} \mathrm{C} \quad(309.6 \mathrm{~K})$. В начальный момент времени температура всей области полагалась равной $T_{0}=36.5^{\circ} \mathrm{C}(309.6 \mathrm{~K})$. Температура на границах области полагалась постоянной и равной $T_{0}$.

Численное решение уравнения (1) выполнено методом конечных разностей $[15,26,27]$ с использованием схемы предиктора-корректора, обеспечивающей второй порядок аппроксимации по времени и пространству $O\left(\tau_{\text {step }}^{2}+h_{\text {step }}^{2}\right)\left(\tau_{\text {step }}, h_{\text {step }}-\right.$ шаги по времени и пространственным координатам) [18].

Для определения областей тепловой абляции тканей в настоящей работе использован подход [28], в соответствии с которым тепловая доза определяется как

$$
t_{D}=\sum_{t=0}^{t_{f i n}} R^{T_{r e f}-T_{m}} \Delta t,
$$

где $t_{D}$ - временной эквивалент тепловой дозы, определяемый в настоящей работе относительно температуры $56^{\circ} \mathrm{C}(329 \mathrm{~K})[3,6,29], T_{m}-$ среднее значение температуры на малом интервале времени $\Delta t$ (в расчетах $\left.\Delta t=\tau_{\text {step }}\right), T_{\text {ref }}=56^{\circ} \mathrm{C}(329 \mathrm{~K})-$ референтное значение температуры, $t_{f i n}-$ конечный момент времени, $R=0.5$ для $T<43^{\circ} \mathrm{C}(316 \mathrm{~K})$ и $R=0.25$ для $T<43^{\circ} \mathrm{C}(316 \mathrm{~K})$. Тепловая доза, необходимая для достижения порога разрушения ткани методом HIFU $t_{56}=1.76 \mathrm{~s}[3,6,29]$, соответствует дозе, определяемой относительно $43^{\circ} \mathrm{C}, t_{43}=240 \mathrm{~min}$, в гипертермии [28].

\section{2. Тепловое воздействие программы HIFU на биоткань}

В настоящей работе рассматривается воздействие на биоткань программы обработки HIFU, состоящей из 31 или 49 импульсов 128-элементного излучателя. Каждый импульс характеризуется трехмерным (осесимметричным) полем интенсивности, определяемым согласно аппроксимации, предложенной в работе [18], и параметрами, приведенными в таблице. Предполагается, что импульсы ультразвукового излучения включаются последовательно и действуют в течение одинакового промежутка времени $t_{H}$ с интервалом $\Delta t_{H}$ между ними. Общее время обработки (действия программы облучения) $t_{s}$ в зависимости от варианта расчета составляет от 2.45 до $77 \mathrm{~s}$. Локализация фокальных точек импульсов в плоскости XY представлена на рис. 2 для программ облучения „спираль Архимеда“ $(a)$ и „квадратная спираль“ (b). В случае применения программы обработки „спираль Архимеда“ последовательность включения импульсов соответствовала подпрограмме „от центра“ (рис. 2,a первой облучается центральная точка 1, последней 31-я точка) или подпрограмме „к центру“ (первой облучалась 31 точка, последней центральная точка). Расстояние по оси $X$ между фокальными точками для случая облучения серией из 31 импульса $h_{x}=2 \mathrm{~mm}$, время наблюдения (расчета) составило $310 \mathrm{~s}$. Для случая облучения серией из 49 импульсов $-h_{x}=0.5 \mathrm{~mm}$, а время наблюдения $25 \mathrm{~s}$.

Параметры импульса (интенсивность в точке фокуса, пространственное распределение интенсивности, длительность), число импульсов в серии $N$, локализация фокальных областей внутри опухоли, порядок включения импульсов и временной интервал между ними, определяют зону теплового разрушения (абляции) биоткани.

Для удобства анализа воздействия серии импульсов на биоткань удобно ввести безразмерные параметры, характеризующие время воздействия и поглощаемую тканью энергию за время действия программы излучения:

$$
\tau_{S}=\frac{t_{S}}{t_{0}}, \quad B y_{S}=\frac{N Q_{H, 0} t_{H}}{c \rho T_{0}} .
$$

Здесь $t_{S}=N t_{H}+(N-1) \Delta t_{H}, Q_{H, 0}-$ удельная мощность теплового источника для центрального импульса 
Параметры программы обработки ткани серией импульсов

\begin{tabular}{c|c|c|c|c|c|c|c|c}
\hline № & 1 & 2 & 3 & 4 & 5 & 6 & 7 & 8 \\
\hline$I_{0}, \mathrm{~W} / \mathrm{cm}^{2}$ & 100 & 100 & 100 & 100 & 100 & 200 & 200 & 250 \\
\hline$t_{H}, \mathrm{~s}$ & 2 & 2 & 2 & 2 & 2 & 2 & 1 & 0.05 \\
\hline$\Delta t_{H}, \mathrm{~s}$ & 0 & 0 & 0 & 0.5 & 0 & 0 & 0 & 0 \\
\hline Программа & $\mathrm{A}<\bullet>$ & $\mathrm{A}>\bullet<$ & $\mathrm{A}<\bullet>$ & $\mathrm{A}<\bullet>$ & $\mathrm{A}<\bullet>$ & $\mathrm{A}<\bullet>$ & $\mathrm{A}<\bullet>$ & $\mathrm{K}<\bullet>$ \\
\hline $\mathrm{N}$ & 31 & 31 & 31 & 31 & 31 & 31 & 31 & 49 \\
\hline$h_{x}, \mathrm{~mm}$ & 2 & 2 & 2 & 2 & 2 & 2 & 2 & 0.5 \\
\hline Учет перфузии & - & - & + & - & - & - & - & \\
\hline$\alpha, \mathrm{m}^{-1}(2 \mathrm{MHz})$ & 14.5 & 14.5 & 14.5 & 14.5 & 9.25 & 9.25 & 14.5 & 9.25 \\
\hline$\lambda, \mathrm{W} /(\mathrm{m} \mathrm{K})$ & 0.21 & 0.21 & 0.21 & 0.21 & 0.21 & 0.21 & 0.42 & 0.21 \\
\hline$\tau_{S}$ & 6.89 & 6.89 & 6.89 & 8.56 & 6.89 & 6.89 & 6.89 & 0.27 \\
\hline Вуз & 2.72 & 2.72 & 2.72 & 2.72 & 1.72 & 3.44 & 2.72 & 0.083
\end{tabular}

Пр имечан и е. $I_{0}-$ интенсивность в точке фокуса „центрального“ импульса (координаты точки фокуса $\left.(0,0,0)\right), t_{H}-$ время импульса, $\Delta t_{H}-$ пауза между импульсами, программа - спираль Архимеда (А) или квадратная $($ К), от центра $<\bullet>$ и к центру $>\bullet<, N-$ число импульсов в серии, $h_{x}$ - расстояние между фокальными точками по оси $X, \alpha-$ коэффициент поглощения, $\lambda-$ коэффициент теплопроводности, $\tau_{S}$, $B y_{S}$ - безразмерные параметры.
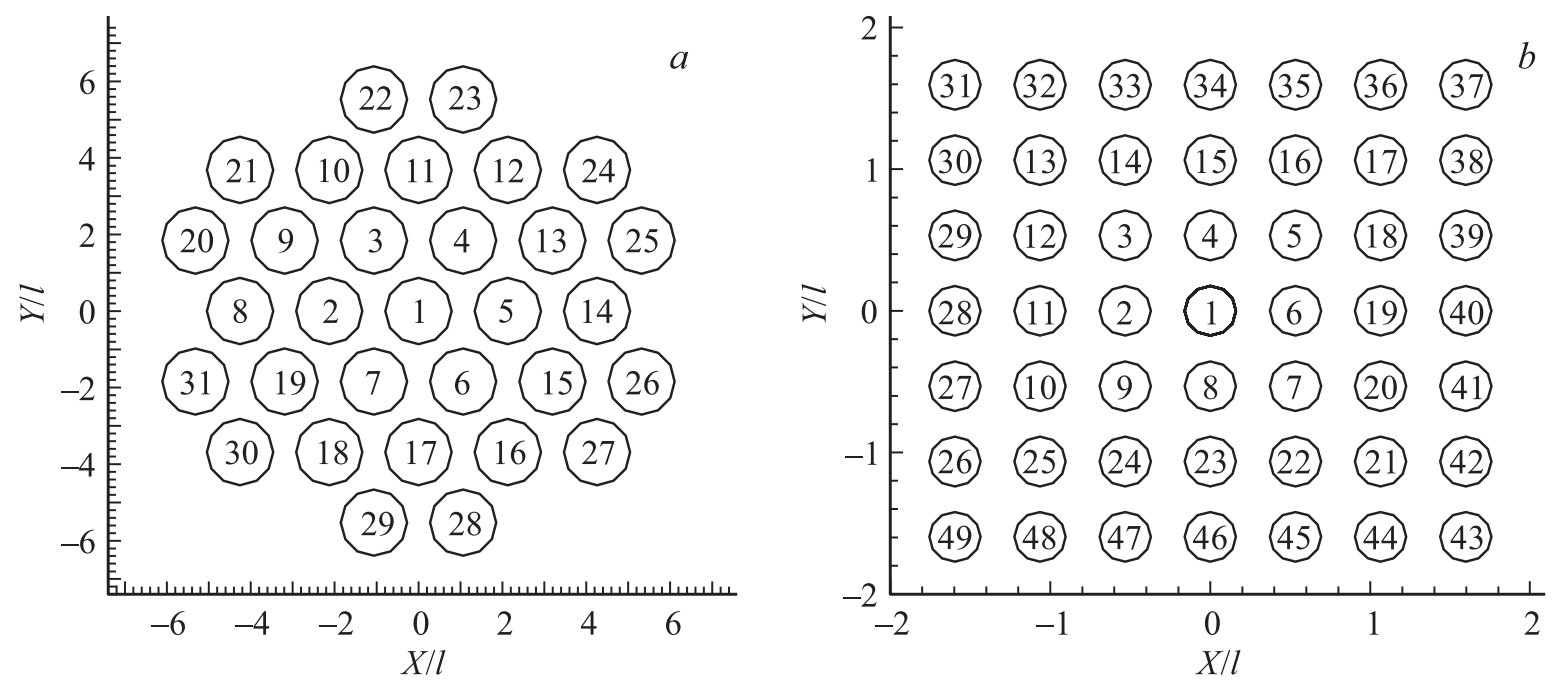

Рис. 2. Пространственное распределение фокальных точек последовательности импульсов. Номер соответствует номеру импульса в серии: $a-$ программа „спираль Архимеда“, $b$ - программа „квадратная спираль“.

в серии с координатами фокуса $(0,0,0)$. Характерный масштаб времени определяется как $t_{0}=c \rho l^{2} / \lambda(l-$ характерный размер). Характерный размер принят равным $l=0.94 \mathrm{~mm}$ и для случая одиночного импульса [18] равен радиусу окружности в плоскости $Z=0$, для точек которой выполняется соотношение $I / I_{F}=0.5\left(I_{F}-\right.$ интенсивность в точке фокуса). Жировой ткани соответствует значение $t_{0} \approx 9 \mathrm{~s}$. Параметры $(5)$ не учитывают процесс перфузии.

Эволюция температуры в центе обрабатываемой области (рис. 3) отражает основные особенности теплового процесса. Максимальная температура централь- ной части для подпрограммы обработки „от центра“ (вариант 1, „спираль Архимеда“) совпадает со случаем одиночного импульса и соответствует времени $t=t_{H} \approx 0.032 t_{S}$. Далее в центральную точку приходят тепловые волны от соседних областей (точек), обрабатываемых в более поздние моменты времени, что соответствует появлению максимумов на температурной кривой 1. Для подпрограммы обработки „к центру“ ситуация иная. Обработка центральной точки происходит последней в серии и соответствует сформировавшемуся температурному полю с $T>T_{0}$. Максимум температуры сдвинут во времени и существенно превышает 

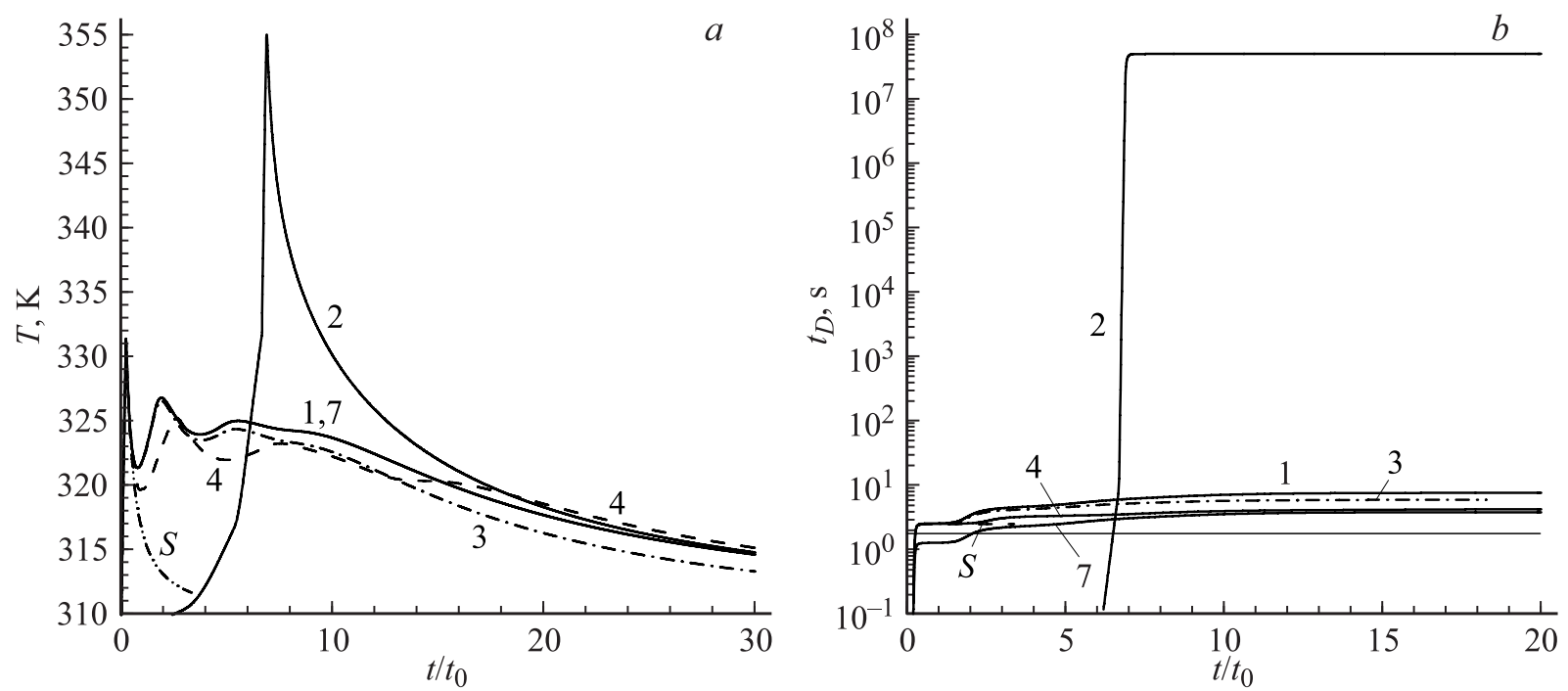

Рис. 3. Эволюция температуры $(a)$ и тепловой дозы $(b)$ в центре области $(0,0,0)$. $S$ - одиночный импульс, цифры - номер варианта согласно таблице. Горизонтальная линия - пороговое значение тепловой дозы $(b)$.

температурный максимум для одиночного импульса. Фактически такой прогрев центральной точки является избыточным с точки зрения концепции „тепловой дозы“, необходимой для разрушения ткани (рис. 3,b). Для программ обработки (1 и 2) общая поглощаемая энергия тканью составляет порядка $86 \mathrm{~J}$ и центр области остывает значительно медленнее случая одиночного импульса (поглощаемая энергия 2.8 J). Несмотря на различие рассматриваемых программ обработки для одинаковой величины энерговклада с течением времени изменение температуры центральной точки становится близким.

Процесс перфузии, учитываемый в варианте расчета 3, приводит к заметному снижению температуры с течением времени при $t / t_{0}>3$ по сравнению с вариантом 1 и, как следствие, уменьшению тепловой дозы, получаемой тканью (рис. 3). Наличие временной задержки $500 \mathrm{~ms}$ между импульсами в варианте расчета 4 также приводит к снижению температуры центральной точки для моментов времени $t / t_{0}<15$. Данный эффект является следствием процесса теплопроводности. Тепловая доза, получаемая тканью, оказывается ниже по отношению к наблюдаемой для варианта 1.

C точки зрения динамики теплового процесса варианты 1 и 7 оказываются подобными. Для них совпадают как безразмерные параметры $\tau_{S}$ и $B y_{S}$, так и ,программа“ обработки (рис. 3,a). Однако величина тепловой дозы, получаемая центральной точкой, является существенно разной (рис.3,$b)$.

На рис. 4 приведены поля температуры для варианта расчета 8 (программа обработки серией импульсов, фокальные точки которых расходятся от центра по „квадратной“ спирали). На момент окончания воздействия программы облучения $\left(t_{S}=2.45 \mathrm{~s}\right)$ поле температур как в поперечной, так и продольной по отношению к направлению распространения ультразву- ка плоскостях имеет сложную форму и не является симметричным (рис. 4, a,c). С течением времени в плоскости $Z=0$ распределение температур становится квазисферическим (рис. 4, $b$ ), в продольной плоскости температурное распределение имеет эллиптический вид (рис. $4, d, t_{S}=25 \mathrm{~s}$ ).

Для программы обработки по спирали Архимеда (вариант расчета 1) на рис. 5 представлены поля температуры в моменты времени $t=t_{S}$, соответствующему окончанию программы обработки (рис. $5, a, b)$ и $t=5 t_{S}$ $($ рис.5, $b, d)$. Для времени $t<t_{S}$ картина нагрева существенным образом зависит от вида подпрограммы обработки („от центра“/„к центру“). Для данного диапазона времени характерна несимметричность температурного поля для всех рассматриваемых вариантов. При этом подпрограмма от центра характеризуется большей степенью асимметрии теплового поля. К моменту времени $t=2 t_{S}$ (на рисунке не приведен) картина распространения тепла размывается и уже нет возможности выделить локализацию фокальных точек. Для времени $t \gg t_{S}$ $\left(t \sim 5 t_{S}\right)$ распространение тепла в плоскости $X Y$ практически не зависит от программы обработки и количества импульсов и приобретает квазисферический характер как в поперечной, так и в продольной плоскостях. Для вариантов 1, 2, 7, характеризующихся одинаковыми безразмерными параметрами $\tau_{S}$ и $B y_{S}$, распределение температур в продольном и поперечном направлениях оказываются близкими (для вариантов расчета 1 и 7 распределения совпадают) (рис. 6). Наличие процесса перфузии приводит к отличию температурных распределений при одинаковых значениях $\tau_{S}$ и $B y_{S}$.

Таким образом, с учетом данных, представленных на рис. 4-6, можно сделать вывод о наличии трех режимов распространения тепла при воздействии программы 

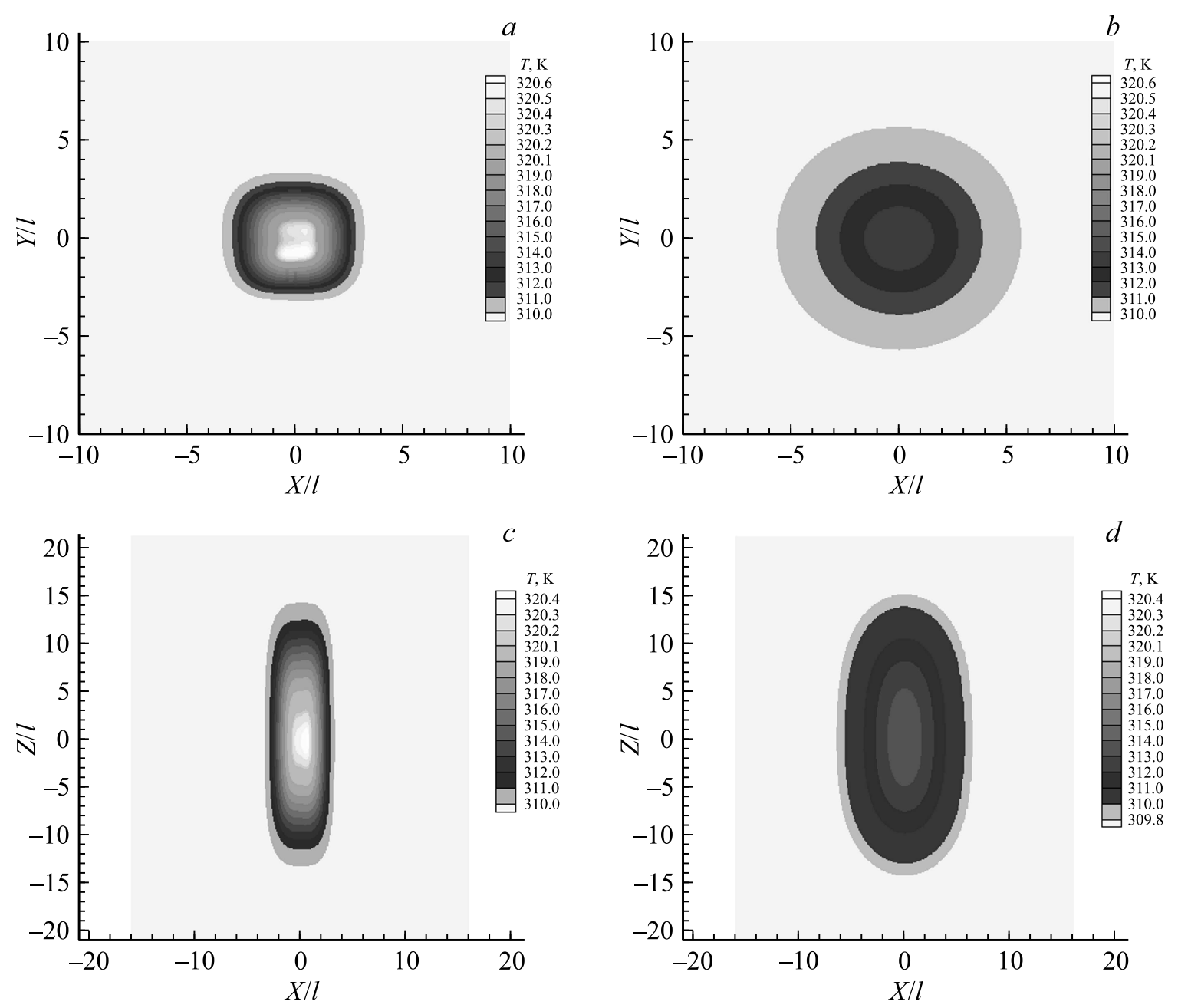

Рис. 4. Поля температуры в плоскостях $Z=0(a, b)$ и $Y=0(c, d)$ (вариант 8). $a, c: t=t_{S}=2.45 \mathrm{~s} ; b, d: t=25 \mathrm{~s}$.

HIFU на биоткань, характеризующихся следующими особенностями:

1) отсутствие симметрии при распространении тепла как в поперечных по отношению к направлению распространения ультразвука плоскостях, так и в продольном направлении. Этот режим характерен для малых времен процесса $t<t_{S}$;

2) в поперечной плоскости распространение тепла носит квазисферический характер, для продольной плоскости данное условие не выполняется;

3) распространение тепла в пространстве носит квазисферический характер с центром в центральной фокальной точке обрабатываемой области. Этот режим достигается при $t \gg t_{S}$.

С учетом „квазисферичности“ полей температуры для режимов (2) и (3) распространение тепла может быть описано аналитическими формулами (распространение тепла в среде от цилиндрического или сферического нестационарного источника). Подбор параметров аналитических моделей зависит не только от режима, но и от значений определяющих параметров, и представляет собой отдельную самостоятельную задачу [30].
Результатом воздействия серии импульсов на ткань является появление области тепловой абляции. Распределение тепловой дозы для варианта 1 в плоскости $Z=0$ приведено на рис. 7. Пространственное распределение тепловой дозы не равномерно. В части области тепловая доза сушественно превышает необходимые для разрушения ткани значения.

Процесс абляции ткани продолжается после завершения программы (серии импульсов) и ,замораживается“ в интервале времени $t_{s}<t<2 t_{S}$. Область тепловой абляции (разрушения ткани) для времени $t=2 t_{S}$ приведена на рис. 8 для вариантов расчета 1 и 2 . Следует обратить внимание на существенное отличие границ области тепловой абляции для подпрограмм „от центра“ и „К центру“(программа „спираль Архимеда“). Для подпрограммы обработки к центру в плоскости $X Y$ существуют зоны неповрежденной ткани, что не допустимо при обработке опухоли. С другой стороны, данная программа обработки позволяет получить большую зону тепловой абляции в продольном направлении. С учетом наличия неповрежденных зон и избыточного, с точки зрения тепловой дозы, перегрева центра области для 

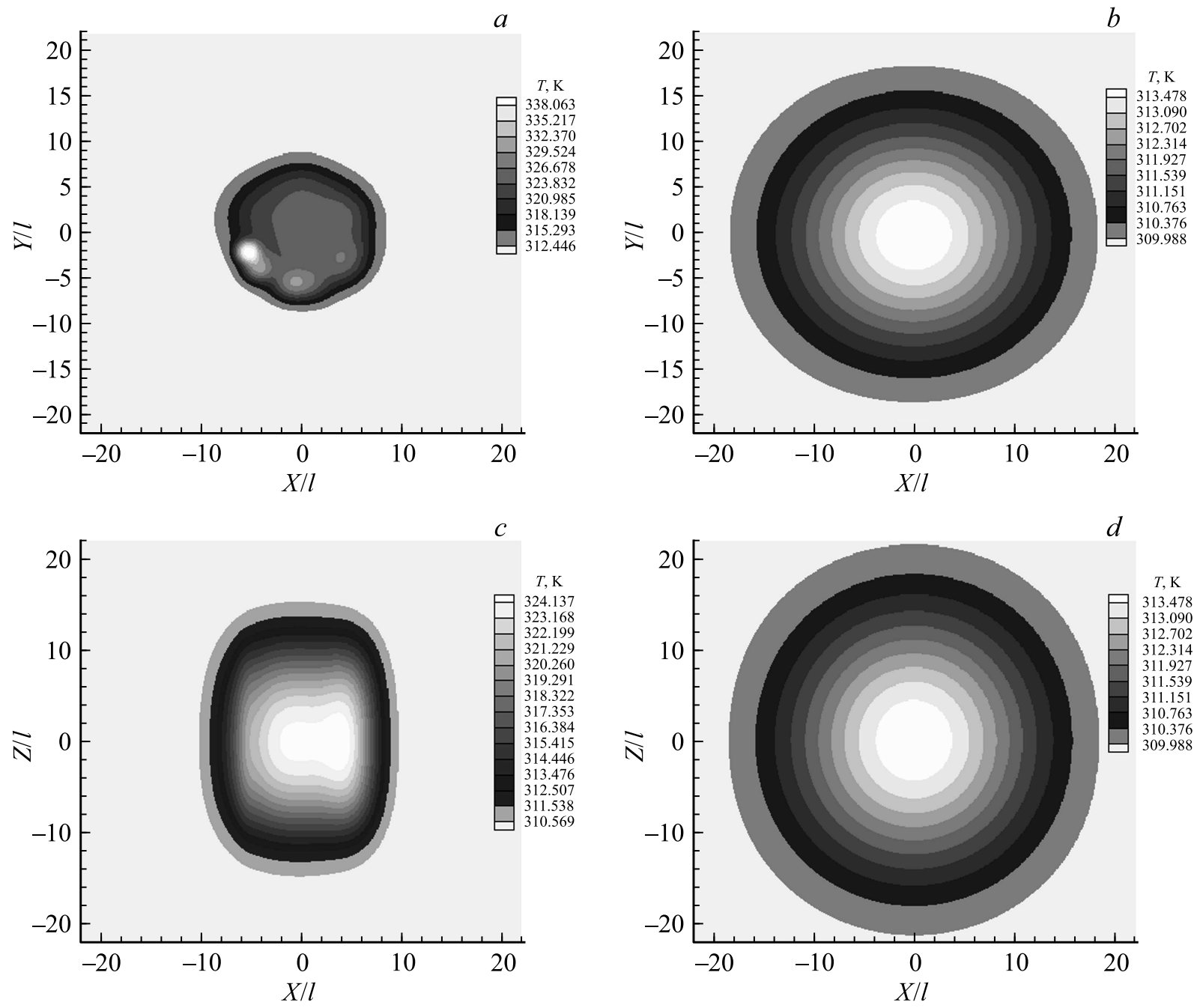

Рис. 5. Поля температуры в плоскостях $Z=0(a, b)$ и $Y=0(c, d)$ (вариант 1). $a, c: t=t_{s}=62 \mathrm{~s} ; b, d: t=5 t_{S}=310 \mathrm{~s}$.
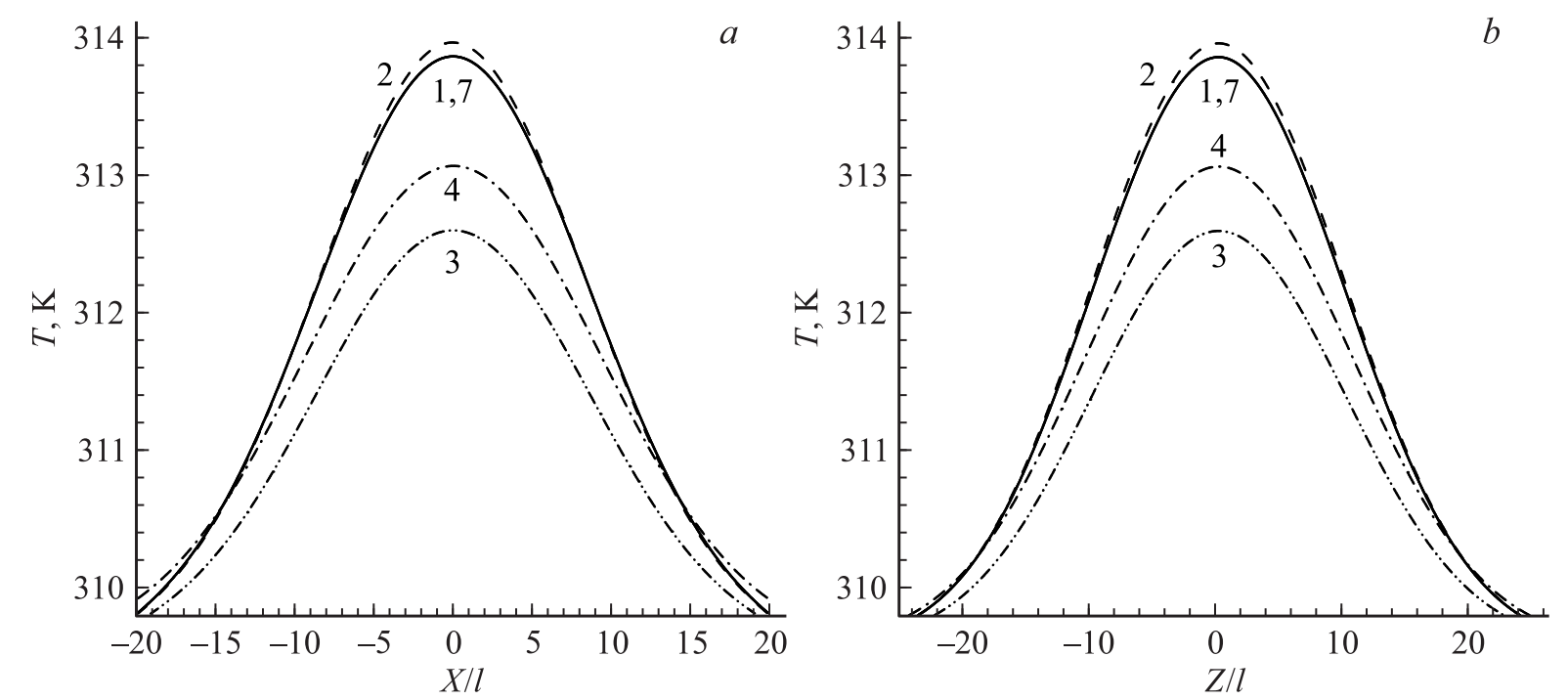

Рис. 6. Распределения температуры в поперечном $(Z=0, Y=0)(a)$ и продольном $(Y=0, X=0)(b)$ направлениях для момента времени $t=5 t_{S}=310 \mathrm{~s}$. Номер кривой соответствует номеру варианта из таблицы. 

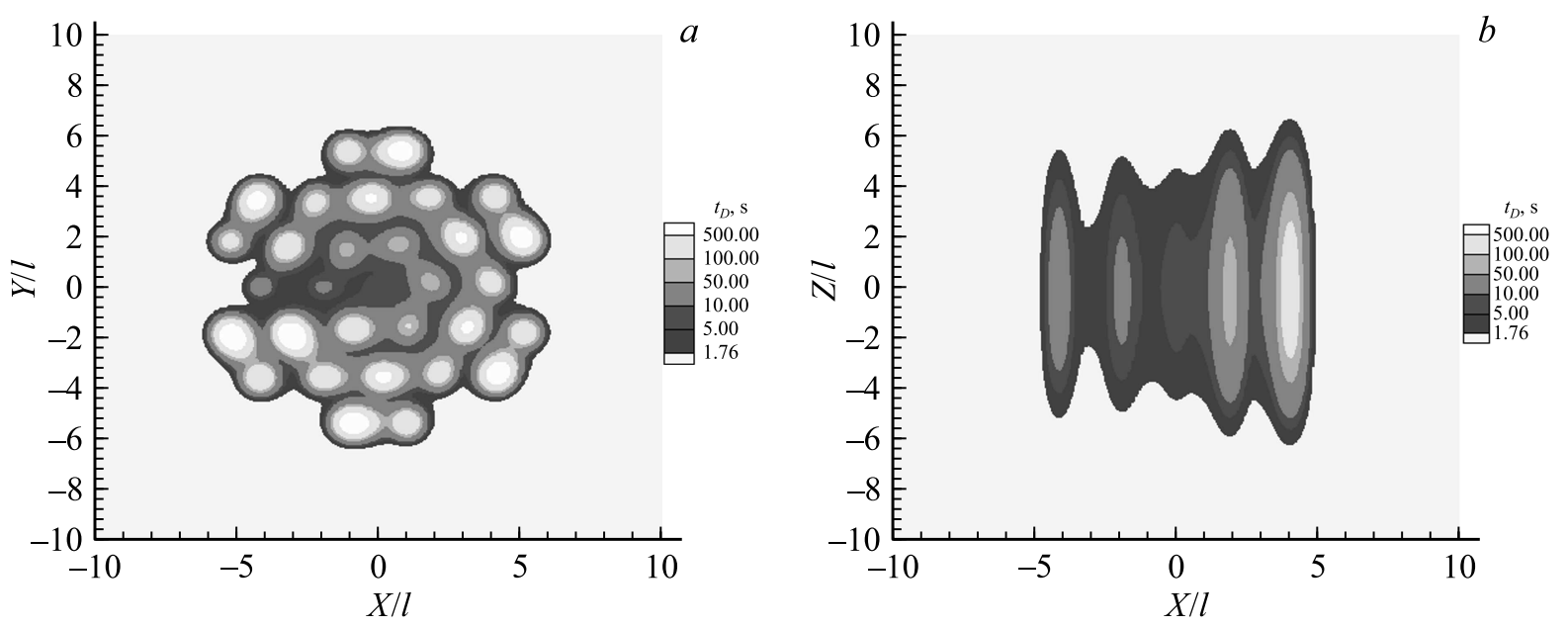

Рис. 7. Распределения тепловой дозы в поперечной плоскости $Z=0(a)$ и в продольной плоскости $Y=0(b)$ для варианта 1 (программа „от центра“).
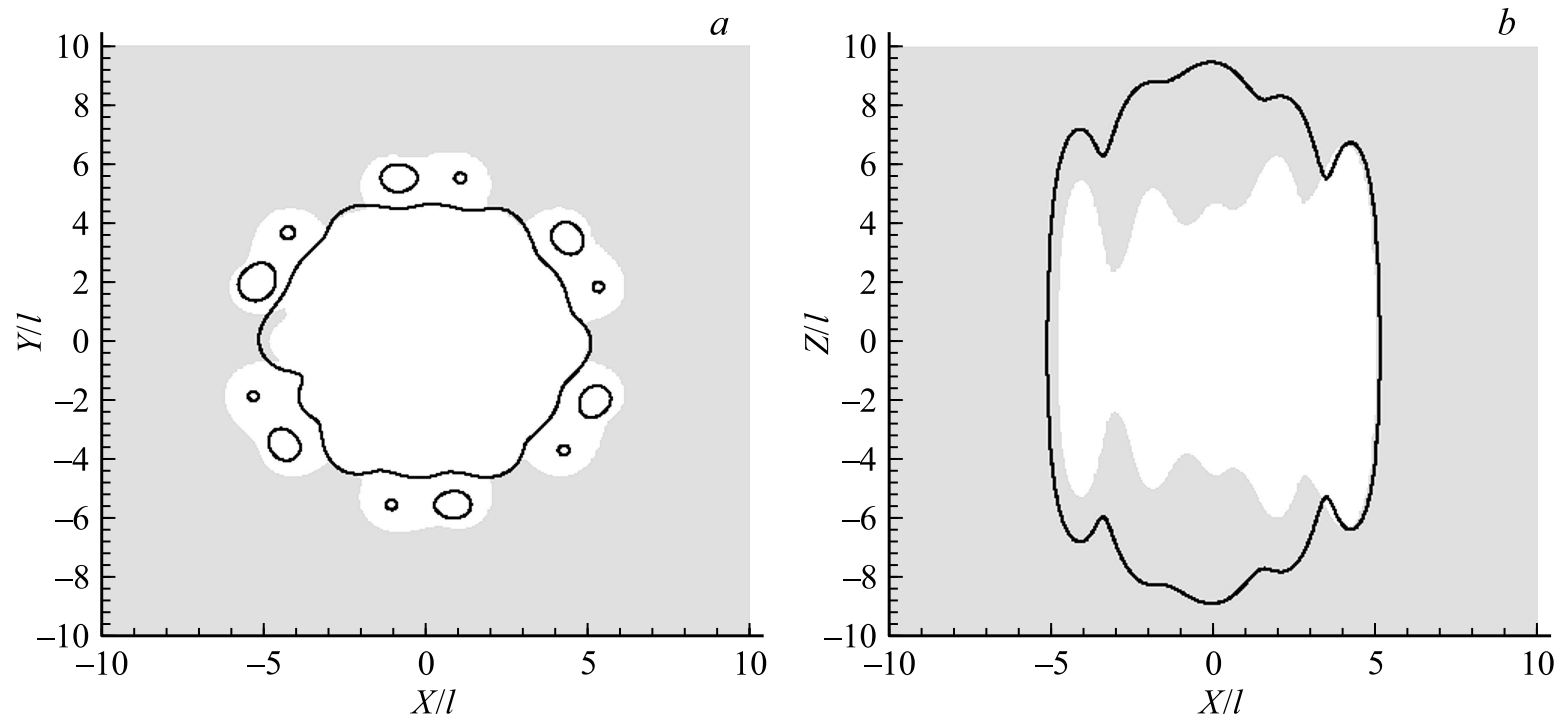

Рис. 8. Граница области поражения в поперечной плоскости $Z=0(a)$ и в продольной плоскости $Y=0(b)$. Контур - вариант 1 („от центра“), линия - вариант 2 („„к центру“).

рассмотренного диапазона параметров подпрограмму „от центра“ следует признать более эффективной.

Полное время наблюдения за изменением температуры в ткани при ее обработке серией из 31 импульса составило $t=5 t_{S}=310 \mathrm{~s}$. На таких достаточно больших временных интервалах на процесс теплопередачи может оказывать влияние процесс перфузии [10]. Для анализа влияния кровотока на распределения параметров был проведен дополнительный расчет 3 (см. таблицу), соответствующий подпрограмме „от центра“. Учет перфузии для жировой ткани (характеризуемой малым коэффициентом объемного расхода крови) приводит к уменьшению температуры центральной точки области (соответствующей геометрическому фокусу), начиная с момента времени $t / t_{S}>3$ (рис. 3 ). К моменту времени $t / t_{S}=30$ различие в температуре центра области для вариантов 1 и 3 составляет $1.5 \mathrm{~K}$. Для указанного момента времени различаются и пространственные распределения температуры. Для рассматриваемого диапазона параметров учет перфузии является крайне важным, так как приводит к появлению неповрежденных участков ткани (рис. 9).

Наличие задержки между импульсами 500 ms также существенно влияет на „сплошность“ области разрушения. Для данного варианта расчета область разрушения содержит разрывы, программа обработки при сохранении длительности импульсов и времени задержки требует коррекции за счет увеличения интенсивности импульсов (рис. 10).

Размеры области абляции для подобных, с точки зрения процесса распространения тепла, вариантов расчета 1 и 7 приведены на рис. 11. Как отмечалось ранее, 

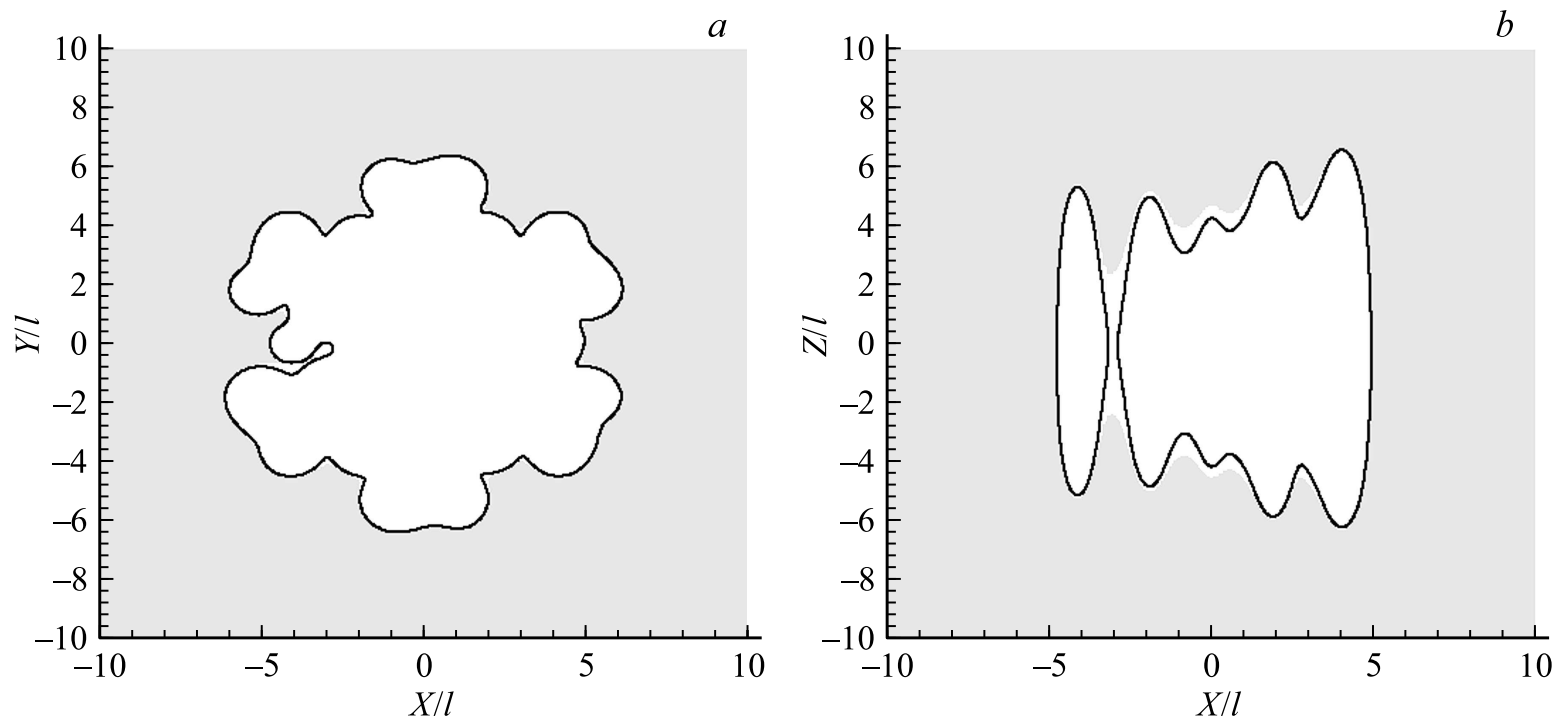

Рис. 9. Граница области поражения в поперечной плоскости $Z=0(a)$ и в продольной плоскости $Y=0(b)$. Контур - вариант 1 , линия - вариант 3 (с учетом перфузии).
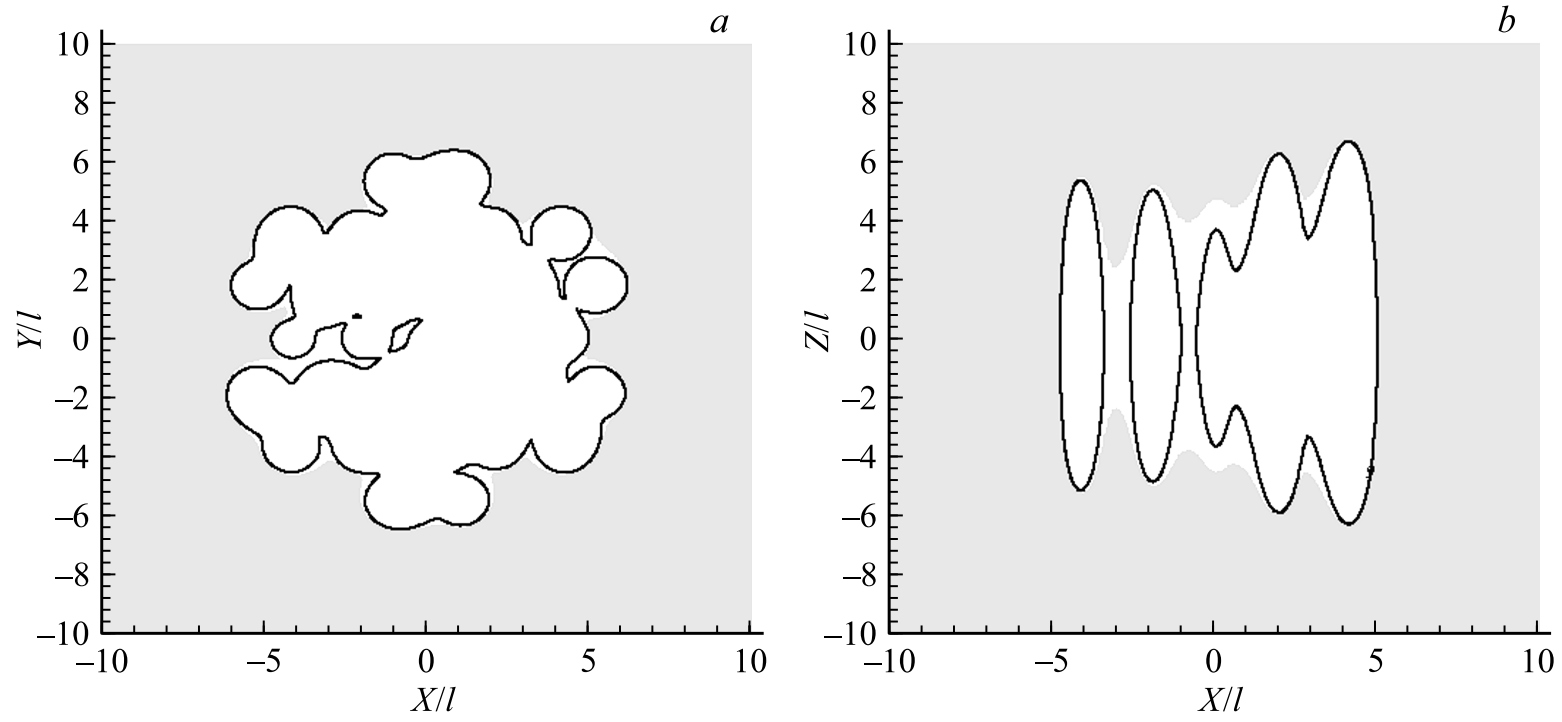

Рис. 10. Граница области теплового поражения в поперечной плоскости $Z=0(a)$ и в продольной плоскости $Y=0(b)$. Контур вариант 1, линия — вариант 4 (с задержкой между импульсами).

тепловая доза для данных вариантов оказывается различной, как следствие, области разрушения различаются. Область абляции для варианта 7 (больший коэффициент теплопроводности, большая интенсивность, меньшее время импульса) содержит непораженные зоны.

Следует отметить существующую неопределенность в оценке коэффициента поглощения жировой ткани [31]. В случае имплементации в расчетах меньшего значения коэффициента поглощения $9.25 \mathrm{~m}^{-1}$ в варианте 5 тепловой дозы, аккумулированной тканью, оказывается недостаточно для формирования области поражения. Область разрушения, близкая по размерам к области абляции, соответствующей варианту 1 , наблюдается при увеличении интенсивности одиночных импульсов в два раза (рис. 12). Таким образом, коэффициент поглощения существенно влияет на результат моделирования области поражения при воздействии серии импульсов HIFU. Положение осложняется тем, что ткань молочной железы может быть неоднородна и содержать фиброгландулярные участки [14]. Поэтому применение расчетных методик для определения области поражения должно сопровождаться персонификацией данных о коэффициенте поглощения тканей пациента и остальных теплофизических параметров.

Имплементация аналитических моделей для квазисферических режимов распространения тепла совместно с термометрией даст возможность оптимизировать алгоритм оценки эффективности медицинских процедур 

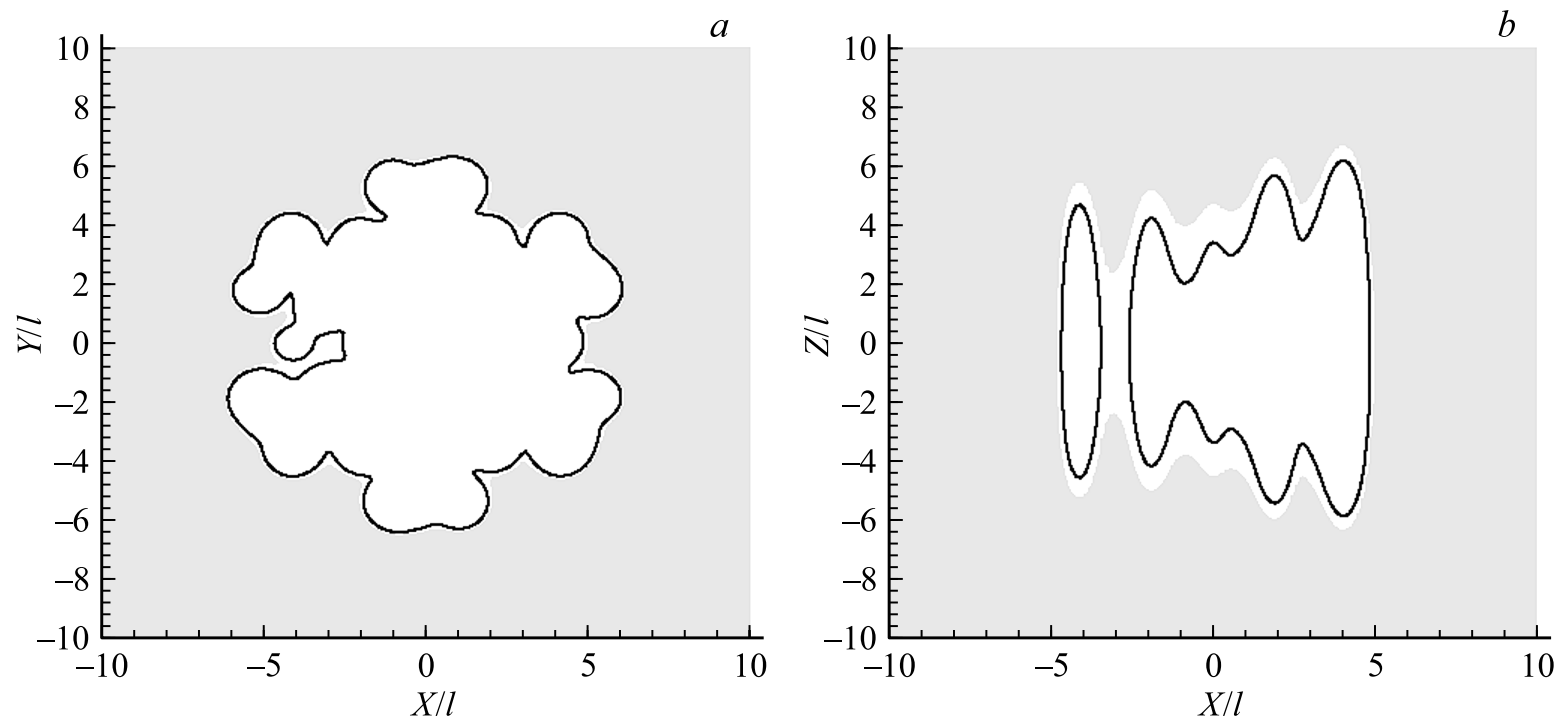

Рис. 11. Граница области теплового поражения в поперечной плоскости $Z=0(a)$ и в продольной плоскости $Y=0(b)$. Контур вариант 1, линия — вариант 7.
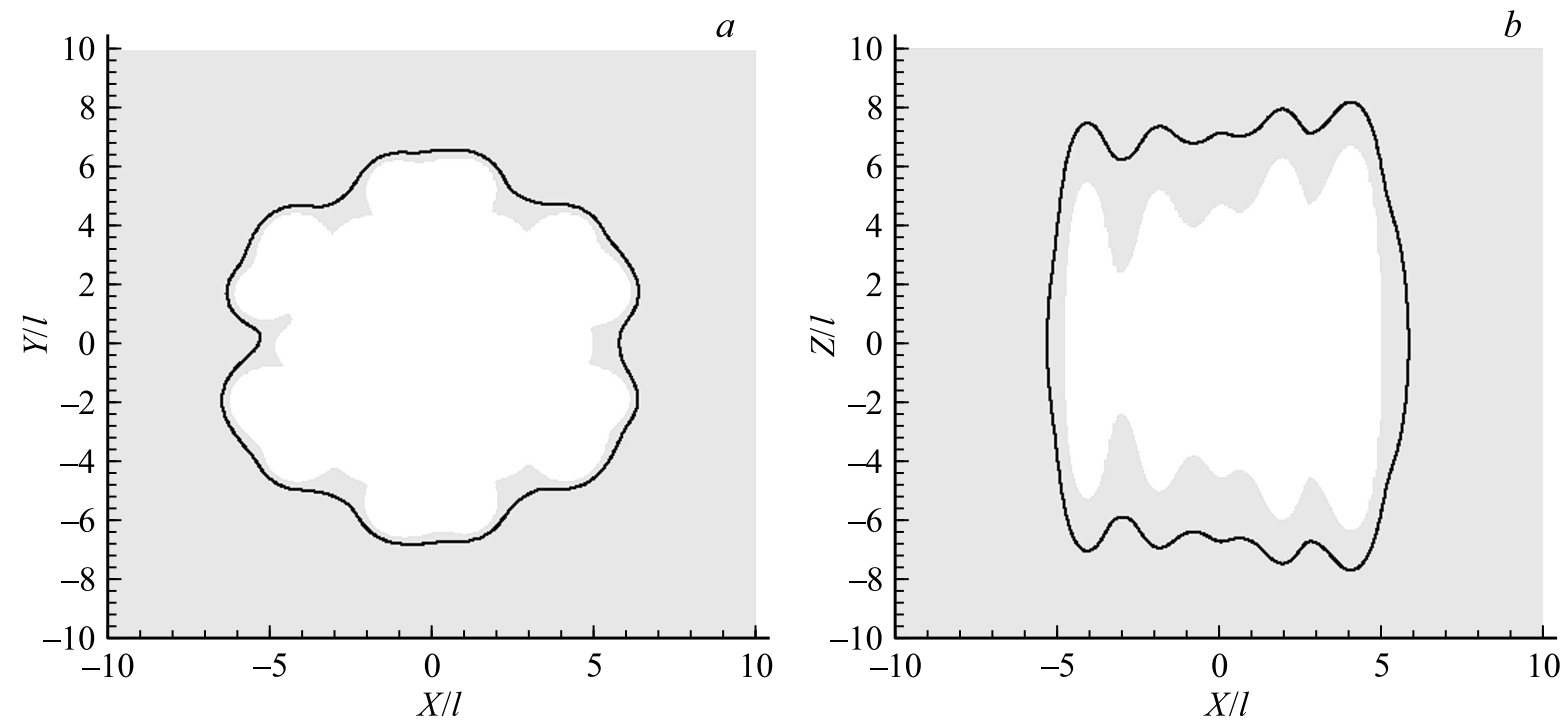

Рис. 12. Граница области теплового поражения в поперечной плоскости $Z=0(a)$ и в продольной плоскости $Y=0(b)$. Контур вариант 1, линия - вариант 6.

HIFU. Возможен следующий протокол обработки опухоли, направленный на „персонификацию“ параметров процедуры:

1) реализация „тестового“ воздействия из 9-16 импульсов в серии с пространственно разнесенными фокальными точками;

2) измерение эволюции температуры в нескольких точках биоткани конкретного пациента (с применением, например, методов ультразвуковой термометрии $[32,33])$;

3) с использованием аналитических моделей и данных термометрии уточнение поглощенной тканью энергии и соответственно коэффициентов поглощения и теплопроводности;
4) численное решение прямой задачи определения теплового поля и области разрушения с уточненным коэффициентом поглощения и варьируемыми параметрами ультразвуковых импульсов. Подбор параметров излучения, необходимый для поражения опухоли с минимальным воздействием на здоровые ткани;

5) использование параметров, полученных в п. 4, в реальной процедуре.

Следует отметить, что использование в п. 1 серии импульсов, а не единичного импульса, позволяет учесть вероятную пространственную неоднородность тканей конкретного пациента. В качестве „тестового“ воздействия могут рассматриваться импульсы ультразвука, 
параметры которых (интенсивность, длительность), не повлекут разрушения ткани.

\section{Заключение}

Воздействие ультразвука высокой интенсивности на биоткань представляет собой сложный феномен, включающий процессы распространения звуковой волны, поглощения и рассеяния ее энергии, теплопереноса, кавитации, химических трансформаций в ткани. В работе основное внимание уделено моделированию процесса распространения тепла на основе численного решения уравнения теплопроводности, описывающего пространственный нестационарный процесс.

Для умеренных интенсивностей ультразвука показана существенная зависимость размеров области разрушения от параметров облучения и наличия перфузии при одинаковой энергии, поглощенной тканью.

Для различных программ HIFU обработки биоткани, характеризующихся как разным количеством импульсов в серии, так и разной пространственно-временной последовательностью импульсов показано установление квазисферического режима распространения тепла на временах, значительно превышающих время воздействия. Наличие квазисферического режима позволяет использовать имеющиеся аналитические модели распространения тепла для описания эволюции температуры и решения обратной задачи - определения теплофизических параметров ткани и прежде всего коэффициента поглощения. Вычисленные таким образом по данным термометрии параметры могут использоваться для оптимизации медицинских протоколов применения HIFU.

\section{Финансирование работы}

Расчеты выполнены с использованием ресурсов СКЦ „Политехнический“. Работа выполнена при финансовой поддержке Министерства науки и высшего образования Российской федерации.

\section{Конфликт интересов}

Авторы заявляют, что у них нет конфликта интересов.

\section{Список литературы}

[1] Izadifar Z., Babyn P., Chapman D. // Ultrasound in Med. Biol. 2017. Vol. 43. N 6. P. 1085-1104.

DOI: 10.1016/j.ultrasmedbio.2017.01.023

[2] Hill C.R., Bamber J.C. Physical principles of medical ultrasonics / TerHaar G.R. (Ed.) NY.: John Wiley \& Sons Ltd., 2004. 528 p.

[3] Гаврилов Л.Р. // Известия ЮФУ. Технические науки. 2013. № 11. C. $208-217$.

[4] Гаврилов Л.Р. Фокусированный ультразвук высокой интенсивности в медицине. М.: Фазис, 2013. 656 с.
[5] Бэйли М.Р., Хохлова В.А., Сапожников О.А., Каргл С.Г., Крам Л.А. // Акуст. журн. 2003. Т. 49. № 4. С. 437-464.

[6] Андрияхина Ю.С., Карзова М.М., Юлдашев П.В., Хохлова В.А. // Акуст. журн. 2019. Т. 65. № 2. С. 147-157. DOI: $10.1134 / \mathrm{S} 0320791919020023$

[7] Wang M., Zhou Y. // Int. J. Hyperthermia. 2016. Vol. 32. N 5. P. 569-582. DOI: $10.3109 / 02656736.2016 .1160154$

[8] Mougenot C., Kohler M.O., Enholm J., Quesson B., Moonen C. // Med. Phys. 2011. Vol. 38. P. 272-282.

[9] Fan X., Hynynen K. // Ultrasound in Med. Biol. 1996. Vol. 22. N 4. P. 471-482. DOI: 10.1016/0301-5629(96)00026-9

[10] Damianou C., Hynynen K. // J. Acoust. Soc. Am. 1994. Vol. 95. N 3. P. $1641-1649$.

[11] Wood A.K.W., Sehgal C.M. // Ultrasound in Med. Biol. 2015. Vol. 41. N 4. P. 905-928. DOI: $10.1016 /$ j.ultrasmedbio.2014.11.019

[12] Филоненко Е.А., Хохлова В.А. // Акуст. журн. 2001. Т. 47. № 4. C. 541-549.

[13] Rosnitskiy P.B., Yuldashev P.V., Sapozhnikov O.A., Maxwell A.D., Kreider W., Bailey M.R., Khokhlova V.A. /I IEEE Trans. Ultrason. Ferroelectr. Freq. Control. 2017. Vol. 64. P. 374-390.

[14] Kohei Okita, Kenji Ono, Shu Takagi, Yoichiro Matsumoto // Int. J. Numer. Meth. Fluids. 2010. Vol. 64. P. 1395-1411.

[15] Самарский А.А., Вабищевич П.Н. Вычислительная теплопередача. М.: Едиториал УРСС, 2003. 784 с.

[16] Meaney P.M., Clarke R.L., Ter Haar G.R., Rivens I.H. // Ultrasound in Med. Biol. 1998. Vol. 24. N 9. P. 1489-1499. DOI: $10.1016 / \mathrm{S} 0301-5629(98) 00102-1$

[17] Филоненко Е.А., Гаврилов Л.Р., Хохлова В.А., Хэнд Д.У. // Акуст. журн. 2004. T. 50. № 2. С. 272-282.

[18] Andreeva T.A., Berkovich A.E., Bykov N.Y., Kozyrev S.V., Lukin A.Ya. // Mater. Phys. Mech. 2019. Vol. 42. P. 625-636. DOI: 10.18720/MPM.4252019_16

[19] Morrison K.P., Keilman G.W., Kaczkowski P.J. // 2014 IEEE Intern. Ultrason. Sympos. Proceed. 2014. P. 400-404.

[20] Pennes H.H. // J. Appl. Phys. 1948. Vol. 1. N 2. P. 93-122.

[21] Stanczyk M., Telega J.J. // Acta of Bioengineering and Biomechanics. 2002. Vol. 4. N 1. P. 31-61.

[22] Physics of Thermal Therapy. Fundamentals and clinical applications / Eduardo G. Moros. (Ed.) Boca Raton: CRC Press, Taylor \& Francis Group, 2012. 375 p.

[23] O’Neil H.T. // J. Acoust. Soc. Am. 1949. Vol. 21. N 5. P. 516-526.

[24] Hasgall P.A., Di Gennaro F., Baumgartner C., Neufeld E., Lloyd B., Gosselin M.C., Payne D., Klingenböck A., Kuster N. „ITIS Database for thermal and electromagnetic parameters of biological tissues“. Version 4.0. May 15. 2018. DOI: 10.13099/VIP21000_04_0.itis.swiss/database

[25] Акопян В.Б., Ершов Ю.А. Основы взаимодействия ультразвука с биологическими объектами М.: МГТУ им. Н.Э. Баумана, 2005. 223 с.

[26] Самарский А.А., Попов Ю.П. Разностные методы решения задач газовой динамики. М.: Наука, 1992. 424 с.

[27] Численное решение многомерных задач газовой динамики / Под ред. С.К. Годунова М.: Наука, 1976. 400 с.

[28] Sapareto S.A., Dewey W.C. // Int. J. Radiation Oncology Biol. Phys. 1984. Vol. 10. P. 787-800.

[29] Yuldashev R., Shmeleva S., Ilyin S., Sapozhnikov O., Gavrilov L., Khokhlova V. // Phys. Med. Biol. 2013. Vol. 58. N 8. P. 2537-2559. DOI: 10.1088/0031-9155/58/8/2537 
[30] Parker K.J. // J. Acoust. Soc. Am. 1985. Vol. 77. N 2. P. 719-725.

[31] Березовский В.А., Колотилов Н.Н. Биофизические характеристики тканей человека. Справочник. Киев: Наукова думка, 1990. 224 с.

[32] Berkovich A.E., Smirnov E.M., Yukhnev A.D., Gataulin Ya.A., Sinitsyna D.E., Tarkhov D.A. // IOPConf. Series: J. Phys.: Conf. Series. 2018. Vol. 1044. P. 012023.

[33] Zhou Y. // Ultrasound Q. 2017 Vol. 33. N 4. P. 253-260.

DOI: $10.1097 /$ ruq.0000000000000300 